\title{
Influence of exposure to tobacco cigarette smoke on the eosinophil count in the nasal mucosa of young patients with perennial allergic rhinitis*
}

\author{
Bertha Beatriz Montaño-Velázquez',Francisco Javier García Vázquez², \\ Ramón Campillo Navarrete ${ }^{3}$, María Dolores Mogica Martínez ${ }^{3}$, \\ Lilia Flores González ${ }^{4}$, Kathrine Jáuregui-Renaud \\ Unidad de Investigación Médica en Otoneurología, CMN sXXI, Instituto Mexicano del Seguro Social, México, D.F. \\ 2Laboratorio de Patología Molecular, Departamento de Anatomía Patológica, Instituto Nacional de Pediatría, S.S., México, D.F. \\ Servicio de Alergia e Inmunología Clínica, Hospital de Especialidades, CMN La Raza, Instituto Mexicano del Seguro Social, \\ México, D.F. \\ ${ }^{4}$ Laboratorio Clínico, Hospital General Regional con UMAA No. 2 Villa Coapa, Instituto Mexicano del Seguro Social, México, D.F.
}

Rhinology 51: 253-258, 2013

DOI:10.4193/Rhino12.190

*Received for publication:

November 9, 2012

Accepted: February 21, 2013

\section{Summary}

Background: To assess the influence of exposure to tobacco cigarette smoke on the eosinophil count and the frequency of apoptosis of eosinophils in the nasal mucosa of teenagers with perennial allergic rhinitis.

Methods: Fifty patients were evaluated (aged 10 to 19 years old): 25 patients with and 25 patients with no recent exposure to tobacco cigarette smoke, by means of The Global Youth Tobacco Survey and cotinine/creatinine ratio. After a clinical evaluation, all the patients replied to a validated questionnaire of the severity of nasal symptoms; then, a nasal sample was processed to identify the eosinophil count and the frequency of apoptosis of eosinophils.

Results: Patients with active exposure to tobacco cigarette smoke had higher eosinophil counts than patients with no exposure to the smoke. In the two groups, apoptosis of eosinophils in the nasal mucosa was scarce and no significant correlation was observed between the frequency/severity of the nasal symptoms and the eosinophil count.

Conclusion: Teenagers with perennial allergic rhinitis and active exposure to tobacco cigarette smoke may show increased eosinophil counts in the nasal mucosa, which might not be related to apoptosis of eosinophils or to the frequency/severity of nasal symptoms.

Key words: allergic rhinitis, tobacco smoke, cigarette, eosinophil count, apoptosis

\section{Introduction}

The World Health Organization has estimated that $40 \%$ of children worldwide are exposed to tobacco smoke at home, and one-quarter of smokers have had their first cigarette before the age of ten ${ }^{(1)}$. Age trends suggest that substance use is a deve- lopmental phenomenon, which increases almost linearly from early to late adolescence ${ }^{(2)}$. Epidemiological studies support that children exposed to environmental tobacco smoke have an increased risk of developing respiratory-tract illnesses. Additionally, allergic rhinitis is a common condition affecting people of 
all ages, with peak lifetime prevalence occurring in teenagers ${ }^{(3)}$.

About $40 \%$ of healthy nonsmokers report a history of rhinitis symptoms associated with exposure to environmental tobacco smoke ${ }^{(4)}$. Experiments in mice indicate that tobacco smoke can elicit a rapid and prolonged exaggerated response with respect to $\lg \mathrm{E}, \lg \mathrm{G} 1$, eosinophils and Th2 cytokines ${ }^{(5)}$, and support its potential to interact with allergen and augment allergic sensitization ${ }^{(6)}$. The effects of tobacco smoke on the upper respiratory airways include the recruitment and activation of inflammatory cells (including eosinophils) which can exacerbate the nasal allergic response ${ }^{(7)}$. Also, irrespective of allergen sensitization, programmed cell death of eosinophils is markedly delayed in atopic diseases ${ }^{(8,9)}$.

Although there are several reports on tobacco smoke exposure as risk factor for having allergic diseases, information available on allergic rhinitis is very scarce. The purpose of this study was to assess the influence of tobacco cigarette smoke exposure on the eosinophil count and the frequency of apoptosis of eosinophils in the nasal mucosa, and their relationship with the frequency/severity of nasal symptoms, in teenagers with perennial allergic rhinitis.

\section{Material and methods}

Ethical considerations

The protocol was approved by the Local Research \& Ethics Committee, and informed consent was obtained from all patients and their parents.

\section{Study population}

Fifty patients with perennial allergic rhinitis participated in the study, all living within the same city area. They were invited to participate consecutively at a public hospital, during their first visit to a specialized allergy clinic. According to exposure to tobacco cigarette smoke, they were classified in two groups, with a similar age (10 to 19 years old), weight, body mass index and men/women ratio:

Group I. Twenty-five patients aged 10 to 19 years old (median 14), 14 were males and 11 were females, with a body mass index from 16.2 to 33.3 (median 21) (one patient was obese). All of them reported exposure to tobacco cigarette smoke by means of The Global Youth Tobacco Survey ${ }^{(10,11)}$, which was confirmed by cotinine/creatinine ratio. Twelve patients reported both active and passive exposure and 13 patients reported only passive exposure. The number of positive allergens during prick testing (AllerStand, Mexico City; IRC guidelines, 1994) was from 1 to 8 (median 3); the most frequent allergens were Dermatophagoides sp (84\%) and house dust mite (16\%). Twenty three of the 25 patients had other atopic diseases concurrent with the rhinitis:
17 had conjunctivitis and dermatitis and 6 had conjunctivitis.

Group II. Twenty-five patients aged 10 to 19 years old (median 13), 16 were male and 9 were females, with a body mass index from 15.8 to 26.9 (median 20.6) (2 patients were obese). None of them reported a history of exposure to tobacco cigarette smoke, by means of The Global Youth Tobacco Survey ${ }^{(10,11)}$, which was confirmed by cotinine/ creatinine ratio. The number of positive allergens during prick testing (AllerStand, Mexico City; IRC guidelines, 1994) was from 1 to 13 (median 2), the most frequent allergens were Dermatophagoides sp (88\%) and house dust mite (24\%). Sixteen of them had concurrent atopic diseases: 3 had conjunctivitis and dermatitis and 13 had conjunctivitis.

Inclusion in the study was considered when perennial allergic rhinitis was diagnosed for the first time and patients had no evidence of infection, sinusitis, otitis media, nasal polyps, anatomical abnormality, systemic disease, lung disease, asthma, atopic dermatitis, seasonal allergic rhinitis or pregnancy; neither they have used immunotherapy, corticosteroids (nasal or systemic), cromolin, anti-inflammatory treatment or antileukotrienes within 3 months prior to participating in the study.

\section{Procedures}

Exposure or no exposure to tobacco cigarette smoke was determined by means of The Global Youth Tobacco Survey ${ }^{(10,11)}$, which includes questions to identify both passive and active exposure to tobacco smoke, and by urinary cotinine/creatinine ratio. On the same day that participants replied to the questionnaire, their urine was collected to measure their cotinine/creatinine ratio ${ }^{(12)}$. Only when the questionnaire and the cotinine/creatinine ratio were consistent, patients were included in the study.

To measure the urinary cotinine/ creatinine ratio, morning urine was collected and frozen at $-20^{\circ} \mathrm{C}$ until analysis, which was performed using solid-phase competitive chemiluminiscent immunoassay for cotinine (Metabolites of Nicotine, DPC France; Immulite 1000, DPC, NJ, USA) and colorimetric Jaffé method for creatinine (Clinical Chemestry IL Test tm Spinreac, Saint Esteve de Bas, Spain; Express Plus, Bayer, Tarrytown, NY, USA). A cut-off value of $21.8 \mathrm{ng} / \mathrm{mg}$ of cotinine/creatinine ratio was used to identify exposure to tobacco smoke ${ }^{(12)}$.

After a clinical evaluation and administration of the short version of the questionnaire from the "International Study of Asthma and Allergies in Childhood" (3), all the patients replied to a validated questionnaire of the severity of nasal symptoms in children with perennial allergic rhinitis ${ }^{(13)}$. The symptoms evaluated were: congestion, sneezing, itching and rhinorrhea. The severity of each symptom was rated by the patient as absent [0], mild [1], moderate [2] or severe [3]. Total symptom score was calculated as the sum of each symptom score (maximum $=12)^{(13)}$. 
Table 1. Mean and standard deviation of cotinine, creatinine and cotinine/ creatinine ratio of 50 patients with perennial allergic rhinitis: 25 with and 25 with no exposure to cigarette tobacco smoke.

\begin{tabular}{|c|c|c|c|}
\hline Variables & $\begin{array}{c}\text { Active } \\
\text { exposure } \\
(n=12)\end{array}$ & $\begin{array}{c}\text { Passive } \\
\text { exposure } \\
(n=13)\end{array}$ & $\begin{array}{c}\text { No } \\
\text { exposure } \\
(n=25)\end{array}$ \\
\hline & Mean, S.D. & Mean, S.D. & Mean, S.D. \\
\hline Age (years) & $16.4,2.5$ & $12.3,1.7$ & $13,2.6$ \\
\hline \multirow[t]{2}{*}{$\begin{array}{l}\text { Body mass } \\
\text { index }\left(\mathrm{kg} / \mathrm{m}^{2}\right)\end{array}$} & $23.9,3.6$ & $20.7,4.7$ & $20.6,3.3$ \\
\hline & Median, Q1-Q3 & Median, Q1-Q3 & Median, Q1-Q3 \\
\hline $\begin{array}{l}\text { Cotinine } \\
\text { (ng) }\end{array}$ & $172,148-387$ & $71,49-91$ & $10,2-10$ \\
\hline $\begin{array}{l}\text { Creatinine } \\
\text { (mg) }\end{array}$ & $1.34,0.6-2.18$ & $1.85,0.84-2.04$ & $0.94,0.75-1.38$ \\
\hline $\begin{array}{l}\text { Cotinine / } \\
\text { creatinine } \\
\text { (ng/mg) }\end{array}$ & $215,78-399$ & $41,29-60$ & $9,5-12$ \\
\hline
\end{tabular}

In a previous study, the administration of the questionnaire to teenagers with perennial allergic rhinitis showed a consistency of 0.89 and repeatability of $96 \%$, with a repeatability coefficient of $2^{(14)}$.

Nasal mucosal specimens were obtained by scraping the middle one-third of inferior turbinates (Rhinoprobe Arlington Scientific Inc, Arlington, TX, USA) and were stained with Wright-Giemsa stain. Then, all samples were analyzed to determine the eosinophil count per squared millimeter, independently by two reviewers, using mouse monoclonal antibodies and mouse mayor basic protein (BMK13, 1:25; Chemicon International, Tamecula, CA, USA), on the slides of twelve calibrated fields (Olympus, BX51, X40) that were randomly selected. Apoptosis of eosinophils was then identified by immunohistochemistry (Caspase-3 rabbit polyclonal antibody, CP229A, 1-100, Biocare Medical, LLC, CA, USA), Fas (CD95, rabbit polyclonal antibody, 1:25, Abcam, Cambridge, UK), anti Bcl-2 (Oncoprotein, mouse monoclonal, 100/D5, 1:50, Biocare Medical, LLC, CA, USA) and TUNEL technique (in situ apoptosis detection kit, Takara, Shiga, Japan).

\section{Statistical analysis}

After Kolmogorov Smirnov test, statistical analysis was performed according to data distribution using " $\mathrm{t}$ " test, " $\mathrm{t}$ " test for proportions, Mann Whitney "U" test, Pearson's correlation coefficient and Analysis of Variance and Tukey Honest Significance Difference for unequal $\mathrm{N}$, values of $\mathrm{p} \leq 0.05$ were considered significant.

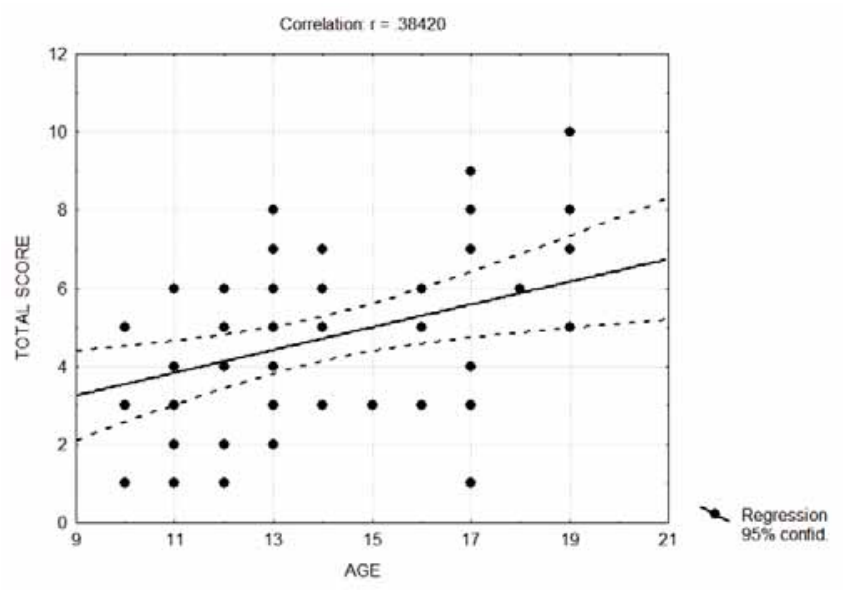

Figure 1. Age versus total score of nasal symptoms of 50 patients with perennial allergic rhinitis: 25 with and 25 with no exposure to cigarette tobacco smoke.

\section{Results}

Clinical characteristics of the patients

Although the two main groups had a similar age, within the group with exposure to tobacco cigarette smoke, the age of the patients was polarized according to the type of exposure, patients with active / passive exposure were significantly older than those with only passive exposure or patients with no exposure (ANOVA \& Tukey HSD for unequal N, p < 0.05), with no difference on their body mass index $(p>0.05)$ (Table 1$)$.

There was no significant difference between the two groups on the positive allergens. However, patients with exposure to tobacco cigarette smoke showed a higher frequency of concurrent atopic diseases than patients with no exposure to tobacco smoke ( $92 \%$ versus $64 \%$, " $t$ " for proportions, $p<0.025$ ). The total symptom score was similar in patients with exposure than in patients with no exposure to tobacco cigarette smoke. Patients with exposure showed scores from 1 to 10 (median 5) and patients with no exposure showed scores from 1 to 8 (median 5) (Mann Whitney, $p>0.05$ ); detailed information has been described in a previous report ${ }^{(15)}$. In patients with and without exposure to tobacco smoke the most frequent symptoms were similar: rhinorrea and sneezing (91\%) in patients with active / passive exposure, rhinorrhea (84\%) in patients with only passive exposure and sneezing (85\%) in patients with no exposure. In the whole group, the total symptom score was related to the age of the patients (Pearson's $r=0.38, p<0.01$ ) (Figure 1 ). 


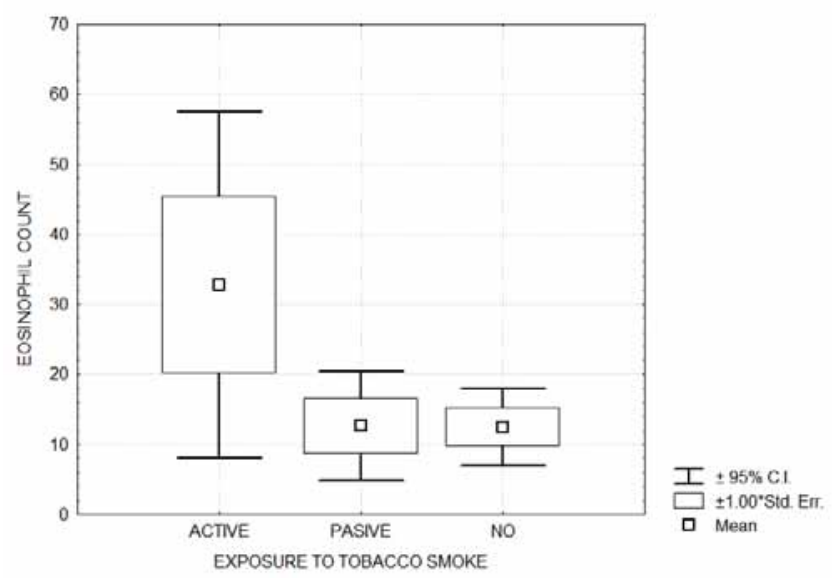

Figure 2. Mean, 95\% Confidence Interval of the mean and Standard Error of the mean of eosinophil count of 50 patients with perennial allergic rhinitis: 25 with and 25 with no exposure to tobacco smoke, according to exposure to cigarette tobacco smoke (active, passive or no exposure).

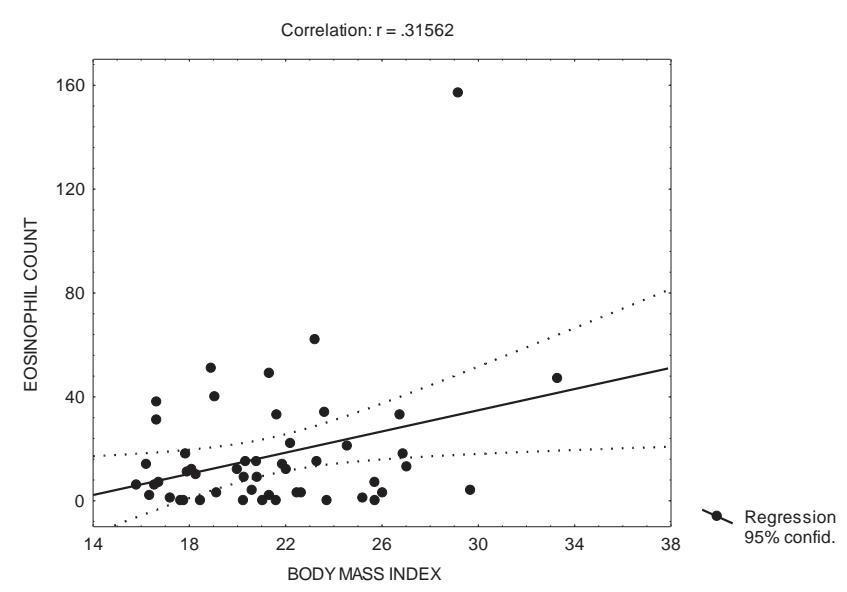

Figure 3. Body mass index versus nasal count of eosinophils on 50 patients with perennial allergic rhinitis: 25 with and 25 with no exposure to cigarette tobacco smoke.
Cotinine/ creatinine ratio

As expected, the urine level of cotinine and the cotinine / creatinine ratio differed between patients with exposure and with no exposure to tobacco cigarette smoke (Kruscall Wallis and Mann Whitney $\mathrm{U}$ tests, $\mathrm{p}<0.01$ ), while the creatinine levels were similar in the two groups. Additionally, within the group of patients with exposure, there was a significant difference on both, cotinine and cotinine / creatinine ratio, between patients reporting active / passive versus those reporting only passive exposure to tobacco cigarette smoke (Mann Whitney $U$ tests, $p$ $<0.01$ ) (Table 1).

\section{Eosinophil count}

Patients with active / passive exposure to tobacco cigarette smoke had more eosinophils than patients with only passive exposure and patients with no exposure to tobacco cigarette smoke (ANOVA \& Tukey HSD for unequal N, p < 0.05), while patients with only passive exposure showed similar counts than patients with no exposure to tobacco smoke (Figure 2). In the whole group, eosinophil count was significantly related to the body mass index of the patients (Pearson's $r=0.31, p<0.05$ ) (Figure 3), but no significant correlation was observed between the eosinophil count and the frequency / severity of the nasal symptoms or the absolute values of the cotinine / creatinine ratio $(p>0.05)$.

\section{Apoptosis of eosinophils}

In the two groups, apoptosis of eosinophils in the nasal mucosa was scarce. It was evident only in two patients with exposure to tobacco cigarette smoke (8\%) and in one patient with no exposure (4\%), with no significant difference between the two groups, or between patients with active / passive versus those with only passive exposure $(p>0.05)$.

\section{Discussion}

This study provides evidence that teenagers with perennial allergic rhinitis who are actively exposed to tobacco cigarette smoke may have an increased count of eosinophils in their nasal mucosa, compared to patients with perennial allergic rhinitis and no exposure. This difference was independent from the frequency of apoptosis of eosinophils on the nasal mucosa and from the frequency / severity of nasal symptoms.

In this study, the evidence of eosinophils in the nasal mucosa with a low frequency of apoptosis in all the patients, is consistent with previous findings in patients with allergic disease of the airways. Since the frequency of apoptosis was low, due to the sample size of the study, the results cannot support or deny a small difference between the groups. However, the finding of an increased eosinophil count in patients who were actively exposed to tobacco smoke, with the highest cotinine / creatinine ratio, with no apparent influence of the frequency of apoptosis support an association between exposure to tobacco smoke and eosinophil attraction in patients with upper airway allergic disease. In animal models and humans, several effects of exposure to tobacco smoke on the immune response have been described ${ }^{(16-19)}$, including modified blood counts of eosinophils and monocytes ${ }^{(20)}$. Studies in patients with asthma have shown that stimulation of eosinophils in airway with IL-5 and eotaxin may play a crucial role in allergic inflammation ${ }^{(21,22)}$, and smoking may influence change in asthmatic airway inflammation by stimulating the production of eotaxins ${ }^{(22)}$. 
Evidence has shown that passive exposure to more than 15 cigarettes per day may cause changes in cellular nasal infiltrates which partly resemble those seen in the nasal mucosa of allergic children ${ }^{(7)}$. In this study, we did not control for the amount of exposure. Though, we observed a difference between patients with active / passive exposure versus patients with only passive exposure, which suggests that, in this study, active smokers had a larger exposure than passive smokers. However, it also has to be considered that active smokers may have a larger absorption of cigarette components than passive smokers. Chemical analytical studies have identified over 3800 compounds in tobacco smoke. Main-stream cigarette smoke is composed of a complex mixture of gases and condensed tar particles (>01 ptm in diameter); side-stream cigarette smoke emissions contain carbon monoxide, ammonia, formaldehyde, benzene, nicotine, acrolein, various gases and particles, and an assortment of potentially genotoxic and/or carcinogenic organic compounds ${ }^{(23)}$. Evidence support that no more than $25 \%$ of the total nicotine content of a cigarette is likely to appear in the main-stream smoke; nevertheless smokers who inhale may absorb up to $90 \%$ of the nicotine in the main-stream smoke drawn into their lungs, while nonsmokers who do not inhale absorb much less nicotine ${ }^{(24)}$.

The age difference between active smokers, while passive smokers had a similar age than no smokers, could be related to the differed frequency of active smoking at different ages, among teenagers ${ }^{(2)}$. On the other hand, the relationship between the total score of nasal symptoms with the age of all participants, is consistent with previous evidence suggesting that children and teenagers with perennial allergic rhinitis frequently underreport their nasal symptoms ${ }^{(25)}$. However, since this study was not designed to assess the trustworthiness of the report of nasal symptoms according to age, we cannot discard that the severity of the disease, more than underreport of symptoms, could have an influence on the results.

The correlation between the eosinophil count and the body mass index, observed in all the patients, support previous evidence showing a relationship between obesity and allergic diseases ${ }^{(26,27)}$, although its influence on upper-airway allergic diseases is still controversial ${ }^{(27)}$.

The finding that exposure to tobacco cigarette smoke was related to a higher frequency of concurrent diseases, is consistent with the epidemiological evidence showing a relationship between exposure to tobacco smoke and the incidence of concurrent atopic diseases ${ }^{(28,29)}$.

The main limitations of this study are the design and the sample size. The cross-sectional design prevents us to discuss any causal relationship; the sample size allowed us to identify only the most evident differences and the larger correlations among the different variables, without denying other possible relationships or differences among the variables of the study.

In conclusion, teenagers with perennial allergic rhinitis and active exposure to tobacco cigarette smoke may show increased eosinophil counts in the nasal mucosa, which might not be related to the frequency of apoptosis of eosinophils.

\section{Acknowledgement}

This study was supported by Fondo de Investigación en Salud, IMSS.

\section{Authorship contribution}

BBMV: Study design and protocol, evaluation of the patients, data base, processing and analysis of samples, interpretation of results, writing and reviewing of the manuscript.

FJGV: Study protocol, processing and analysis of samples, interpretation of results, reviewing of the manuscript.

RCN, MDMM and LFG: Study protocol, evaluation of the patients, data collection, reviewing of the manuscript.

KJR: Study design and protocol, data base, data analysis, interpretation of results, writing and reviewing of the manuscript.

\section{Conflict of interest}

The authors declare no conflict of interest.

\section{References}

1. World Health Organization. Tobacco Free Initiative. The Tobacco Atlas 2002. Available at http://www.who.int/tobacco/resources/ publications/tobacco_atlas/en/. Accessed January 30, 2011

2. Young SE, Corley RP, Stallings MC, Rhee SH, Crowley TJ, Hewitt JK. Substance use, abuse and dependence in adolescence: prevalence, symptom profiles and correlates. Drug Alcohol Depend. 2002; 68: 309-322.

3. Asher MI, Montefort S, Björkstén B, Lai CK, Strachan DP, Weiland SK, Williams H. ISAAC
Phase Three Study Group. Worldwide time trends in the prevalence of symptoms of asthma, allergic rhinoconjunctivitis, and eczema in childhood: ISAAC Phases One and Three repeat multicountry cross-sectional surveys. Lancet. 2006; 368, 733-743.

4. Bascom R, Kulle T, Kagey-Sobotka A, Proud D. Upper respiratory tract environmental tobacco smoke sensitivity. Am Rev Respir Dis. 1991; 143: 1304-1311.

5. Seymour BWP, Pinkerton KE, Friebertshauser KE, Coffman RL, Gershwin LJ. Secondhand smoke is an adjuvant for T-helper-2 responses in a murine model of allergy. J Immunol. 1997; 159: 6169-6175.

6. Rumold R, Jyrala M, Diaz-Sanchez D. Second hand Smoke Induces Allergic Sensitization in Mice. J Immunol. 2001; 167: 4765-4770.

7. Vinke JG, KleinJan A, Severijnen WM, Fokkens WJ. Passive smoking causes an "allergic" cell infiltrate in the nasal mucosa of non-atopic children. Int J Pediatr Otorhinolaryngol. 1999; 51: 73-81.

8. Wedi B, Raap U, Lewrick H, Kapp A. Delayed eosinophil programmed cell death in vitro: A common feature of inhalant allergy and 
extrinsic and intrinsic atopic dermatitis. J Allergy Clin Immunol. 1997; 100: 536-543.

9. $\mathrm{Xu} J$, Jiang $F$, Nayeri F, Zetterström $O$. Apoptotic eosinophils in sputum from asthmatic patients correlate negatively with levels of IL-5 and eotaxin. Respir Med. 2007; 101: 1447-1454.

10. Warren $C W$, Jones NR, Peruga A, et al. Global youth tobacco surveillance, 2000-2007. Morbidity and Mortality Weekly Report. Surveillance Summaries (Washington, D.C. 2002) 2008, 57 (1): 1-28

11. Warren CW. Tobacco use among youth: a cross country. Tob Control. 2002; 11: 252270.

12. Olivieri M, Bodini A, Peroni DG, et al. Passive smoking in asthmatic children. Effect of a "smoke-free house" measured by urinary cotinine levels. Allergy Asthma Proc. 2006; 27: 350-353.

13. Corren J, Harris AG, Aaronson D, et al. Efficacy and safety of loratadine plus pseudoephedrine in patients with seasonal allergic rhinitis and mild asthma. J Allergy Clin Immunol. 1997; 100: 781-788.

14. Montaño Velazquez BB, Jáuregui-Renaud $K$ Campillo Navarrete $M R$, et al. Evaluation of a questionnaire for measuring nasal symptoms in subjects with allergic rhinitis. Rev Alerg Mex. 2003: 50: 17-21.

15. Montaño-Velázquez BB, Navarrete $R$, Mogica Martínez M, Becerril-Angeles M, Jáuregui-Renaud K. Rhinomanometry in young patients with perennial allergic rhinitis with/without recent exposure to tobacco smoke. Clin Otolaryngol. 2011; 36 320-324

16. Kalra $R$, Singh $S P$, Savage $S M$, Finch $G L$, Sopori ML. Effects of Cigarette smoke on the immune response: chronic exposure to cigarette smoke impairs antigen-medi- ated signaling in T cells and depletes IP3 sensitive calcium stores. J Pharmacol Exp Ther. 2000; 293: 166-171.

17. Matsumoto K, Aizawa H, Inoue H, Koto H, Takata S, Shigyo M, Nakano H, Hara N. Eosinophilic airway inflammation induced by repeated exposure to cigarette smoke. Eur Respir J. 1998; 12: 387-394.

18. Mio T, Romberger DJ, Thompson AB, et al. Cigarette smoke induces interleukin-8 release from human bronchial epithelial cells. Am J Resp Crit Care Med. 1997; 155: 1770-1776.

19. Sopori ML, Kozak W, Savage SM, Geng Y, Kluger MJ. Nicotine-induced modulation of $T$ cell function. Implications for inflammation and infection. Adv Exp Med Biol. 1998; 437: 279-289

20. Jensen E J, Pedersen B, Narvestadt E, Dah R. Blood eosinophil and monocyte counts are related to smoking and lung function. Respir Med. 1998; $92: 63-69$.

21. Xu J, Jiang F, Nayeri F, Zetterström $O$ Apoptotic eosinophils in sputum from asthmatic patients correlate negatively with levels of IL-5 and eotaxin. Respir Med. 2007 101: 1447-1454

22. Krisiukeniene A, Babusyte A, Stravinskaite K, Lotvall J, Sakalauskas R, Sitkauskiene B. Smoking affects eotaxin levels in asthma patients. J Asthma. 2009; 46: 470-476.

23. Dye JA, Adler KB. Effects of cigarette smoke on epithelial cells of the respiratory tract. Thorax. 1994; 49: 825-834

24. Armitage AK, Dollery CT, Houseman TH, Lewis PJ, Turner DM. Absorption and metabolism of nicotine from cigarettes. BMJ. 1975: 4: 313-316.

25. Priftisa KN, Drigopoulosb K, Sakalidoua A, Trigaa M, Kallisb V, Nicolaidouc P. Subjective and objective nasal obstruction assessment in children with chronic rhinitis. Int J Pediatr Otorhinolaryngol. 2006; 70: 501-505.

26. Bråbäck L, Hjern A, Rasmussen F. Body mass index, asthma and allergic rhinoconjunctivitis in Swedish conscripts - a national cohort study over three decades. Respir Med. 2005; 99: 1010-1014.

27. Nagel G, Koenig W, Rapp K, Wabitsch M Zoellner I, Weiland SK. Associations of adipokines with asthma, rhinoconjunctivitis, and eczema in German schoolchildren. Pediatr Allergy Immunol. 2009: 20: 81-88.

28. Polosa R, Knoke JD, Russo C, et al. Cigarrete smoking is associated with a great risk of incident asthma in allergic rhinitis. J Allergy Clin Immunol. 2008; 121: 1428-1434.

29. Demir AU, Celikel S, Karakaya G, Kalyoncu AF. Asthma and allergic diseases in school children from 1992 to 2007 with incidence data. J Asthma. 2010; 47: 1128-1135.

Dra. Kathrine Jáuregui-Renaud Unidad de Investigación Médica en Otoneurología P.B. Edificio C Salud en el Trabajo Centro Médico Nacional sXXI, IMSS Av. Cuauhtémoc 330 Colonia Doctores, C.P. 06720 México D.F.

Tel/Fax: +5255-56276900 ext. 21669 E-mail:

kathrine.jauregui@imss.gob.mx

\section{1th INTERNATIONAL COURSE IN ADVANCED SINUS SURGERY TECHNIQUES}

\section{Dissection course with fresh frozen cadaver heads}

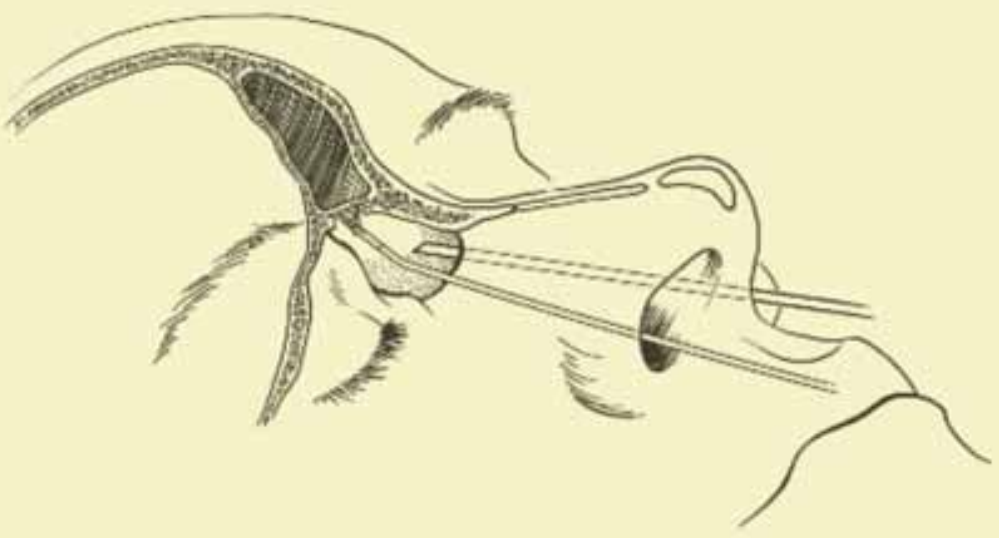

Teacher of Honour: Robert C Kern, MD

March 27-28, 2014

For further information contact Wytske J. Fokkens, MD, PhD ENT dept. AMC Course Secretariat

Tel: 0031205668586 / Fax 0031205669573

Email:m.b.vanhuiden@amc.uva.nl

Department of Otorhinolaryngology Academic Medical Center of the University of Amsterdam

The Netherlands 